\title{
CONVECTIVE FLOW OF MICROPOLAR FLUIDS ALONG AN INCLINED Flat Plate WITH VARIABLE ELECTRIC CONDUCTIVITY AND UNIFORM SURFACE HEAT FLUX
}

\author{
Md. Jashim Uddin \\ Faculty of Science and Information Technology, Daffodil International University, 102 Shukrabad, \\ Mirpur Road, Dhaka-1207. \\ E-mail: jas_fluidm@yahoo.com
}

\begin{abstract}
Magnetohydrodynamic (MHD) twodimensional steady convective flow and heat transfer of micropolar fluids flow along an inclined flat plate with variable electric conductivity and uniform surface heat flux has been analyzed numerically in the presence of heat generation. With appropriate transformations the boundary layer partial differential equations are transformed into nonlinear ordinary differential equations. The local similarity solutions of the transformed dimensionless equations for the velocity flow, microrotation and heat transfer characteristics are assessed using NachtsheimSwigert shooting iteration technique along with the sixth order Runge-Kutta-Butcher initial value solver. Numerical results are presented graphically in the form of velocity, microrotation, and temperature profiles within the boundary layer for different parameters entering into the analysis. The effects of the pertinent parameters on the local skin-friction coefficient (viscous drag), plate couple stress and the rate of heat transfer (Nusselt number) are also discussed and displayed graphically.
\end{abstract}

Keywords: Convective flow, Micropolar fluid, Heat transfer, Electric conductivity, Inclined plate and Locally self-similar solution

\section{Nomenclature}

$B_{0} \quad$ uniform magnetic field

$C_{f} \quad$ skin-friction coefficient

$C_{p} \quad$ specific heat at constant pressure

$g_{0} \quad$ gravitational acceleration

$g$ dimensionless microrotation

$h(x) \quad$ local heat transfer coefficient

j micro-inertia density

M magnetic field parameter

$M_{x} \quad$ dimensionless couple stress

$M_{w} \quad$ couple stress at the plate

$\mathrm{Nu}{ }_{x} \quad$ Nusselt number

Pr Prandtl number

\begin{tabular}{ll}
$Q_{0}$ & heat source parameter \\
$Q$ & non-dimensional heat generation parameter \\
$q_{w}$ & surface heat flux \\
$S$ & microrotation coupling coefficient \\
$T$ & temperature within boundary layer \\
$T_{w}$ & temperature at the plate \\
$T_{\infty}$ & temperature of the ambient fluid \\
$u$ & velocity along $X$-axis \\
$v$ & velocity along $y$-axis \\
$X$ & coordinate along the plate \\
$y$ & coordinate normal to the plate \\
Greek & Symbols \\
$\alpha$ & angle of inclination \\
$\beta$ & coefficient of volume expansion \\
$\gamma$ & local buoyancy parameter \\
$\rho$ & density of the fluid \\
$\mu$ & coefficient of dynamic viscosity \\
$v$ & apparent kinematic viscosity \\
$v_{s}$ & spin-gradient viscosity \\
$\Delta$ & vortex viscosity parameter \\
$\xi$ & micro-inertia density parameter \\
$\sigma$ & microrotation component \\
$\sigma$ & magnetic permeability \\
$\kappa$ & thermal conductivity \\
$\eta$ & similarity parameter \\
$\theta$ & dimensionless temperature \\
$\Delta \eta$ & step size \\
\hline &
\end{tabular}

1. Introduction

Many transport processes occurring both in nature and in industries involve fluid flows with the combined heat and mass transfer. Such flows are driven by the multiple buoyancy effects arising from the density variations caused by the variations in temperature as well as species concentrations. Micropolar fluids are those, which contain micro-constituents that can undergo rotation, the presence of which can affect the hydrodynamics of the flow so that it can be distinctly non-Newtonian. These fluids are fluids with microstructure belonging to a 
class of complex fluids with nonsymmetrical stress tensor referred to as micromorphic fluids. It has many practical applications, for examples analyzing the behavior of exotic lubricants, the flow of colloidal suspensions or polymeric fluids, liquid crystals, additive suspensions, animal blood, body fluids, and turbulent shear flows. Ariman et al. [1] has given an excellent review of micropolar fluids and their applications. Hoyt and Fabula [2] have shown experimentally that the fluids containing minute polymeric additives indicate considerable reduction of the skinfriction (about 25-30\%), a concept that can be well explained by the theory of micropolar fluids.

Convective flow over a flat plate that is immersed in a micropolar fluid has attracted an increasing amount of attention since the early studies of Eringen [3]. Since then many investigators have studied and reported results for micropolar fluids of whom the names of Ebert [4], Jena and Mathur [5], Soundalgekar and Takhar [6], Gorla and Takhar [7], Yucel [8], Gorla [9], Khonsari and Brew [10], Khonsari [11], Gorla et al. [12], Gorla and Nakayama [13], Char and Chang [14], Raptis [15] are worth. Rahman and Sattar [16] studied transient convective flow of micropolar fluid past a continuously moving vertical porous plate in the presence of radiation. Very recently Rahman and Sultana [17] studied radiative heat transfer flow of micropolar fluid past a vertical porous flat plate with uniform plate temperature as well as variable surface heat flux in a porous medium.

Molla et al. [18] studied natural convection flow along a heated wavy surface with a distributed heat source as given in Vajravelu Hadjinolaou [19]. Mohammadein and Gorla [20] investigated heat transfer in a micropolar fluid over a stretching sheet with viscous dissipation and internal heat generation. Rahman and Sattar [21] studied Magnetohydrodynamic convective flow of a micropolar fluid past a continuously moving vertical porous plate in the presence of heat generation / absorption.

Alam et al. [22] studied MHD free convective heat and mass transfer flow past an inclined surface with heat generation. Alam et al. [23] studied effects of thermophoresis and chemical reaction on unsteady hydromagnetic free convection and mass transfer flow past an impulsively started infinite inclined porous plate in the presence of heat generation/absorption. Very recently Alam et. Date of submission : 28.04.2009 Date of acceptance : 12.12.2010
Al. [23] studied the effects of variable suction and thermophoresis on steady MHD combined free-forced convective heat and mass transfer flow along a semi-infinite permeable inclined plat in the presence of thermal radiation. Therefore in the present study, we have extended the work of Rahman and Sattar [20] for micropolar fluid to an impermeable inclined plate considering variable electric conductivity in the presence of uniform surface heat flux. The resulting governing equations are solved by Nachtsheim-Sweigert shooting iteration technique together with Runge-Kutta-Butcher initial value solver and reported graphically. Finally, numerical results for the local skinfriction coefficient, local couple stress and local Nusselt number are presented graphically for different values of the material parameters.

\section{Governing Equations of the Flow}

Let us consider a steady two-dimensional MHD laminar convective flow of viscous, incompressible micropolar fluids along a semi-infinite inclined permeable flat plate with an acute angle $\alpha$ to the vertical. The magnetic field is assumed to be in the $y$ direction and is defined as: $\overrightarrow{\mathrm{B}}=(0, B(x))$. (1)

Let us consider the Cartesian coordinates $(x, y)$ introduced for the description of the Magnetohydrodynamic flow. The flow configurations and the coordinate system are shown in the following Fig.1.

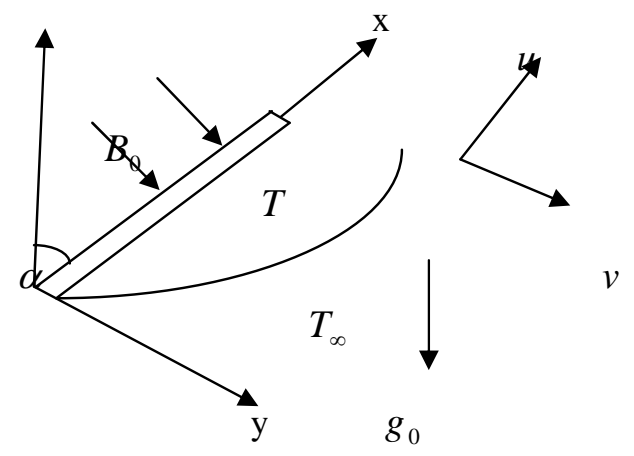

Fig.1: Flow configurations and the coordinate system.

Within the framework of the above-noted assumptions the convective flow of steady incompressible micropolar fluids subject to the Boussinesq approximation for the flow and heat transfer can be written in the following form: 
Continuity Equation:

$\frac{\partial u}{\partial x}+\frac{\partial v}{\partial y}=0$,

(2)

Momentum Equation:

$u \frac{\partial u}{\partial x}+v \frac{\partial u}{\partial y}=\left(v+\frac{S}{\rho}\right) \frac{\partial^{2} u}{\partial y^{2}}+\frac{S}{\rho} \frac{\partial \sigma}{\partial y}+$

$g_{0} \beta\left(T-T_{\infty}\right) \operatorname{Cos} \alpha-\frac{\sigma_{0}^{\prime}(B(x))^{2} u}{\rho}$,

Angular Momentum Equation:

$u \frac{\partial \sigma}{\partial x}+v \frac{\partial \sigma}{\partial y}=\frac{v_{s}}{\rho j} \frac{\partial^{2} \sigma}{\partial y^{2}}-\frac{S}{\rho j}\left(2 \sigma+\frac{\partial u}{\partial y}\right)$,

\section{Energy Equation:}

$u \frac{\partial T}{\partial x}+v \frac{\partial T}{\partial y}=\frac{k}{\rho c_{p}} \frac{\partial^{2} T}{\partial y^{2}}+\frac{Q_{0}}{\rho c_{p}}\left(T-T_{\infty}\right)$,

where $u, v$ are the velocity components along

$x, y$ co-ordinates respectively, $v=\frac{\mu}{\rho}$ is the

kinematic viscosity, $\rho$ is the mass density of the fluid, $\mu$ is the coefficient of dynamic viscosity, $v_{s}=\left(\mu+\frac{S}{2}\right) j \quad$ [27] is the microrotation viscosity or spin-gradient viscosity, $S$ is the microrotation coupling coefficient (also known as the coefficient of gyro-viscosity or as the vortex viscosity), $\sigma$ is the microrotation component normal to the $x y$-plane, $j$ is the micro-inertia density, $T$ is the temperature of the fluid in the boundary layer, $T_{\infty}$ is the temperature of the fluid outside the boundary layer, $c_{p}$ is the specific heat of the fluid at constant pressure, $k$ is the thermal conductivity, $g_{0}$ is the acceleration due to gravity, $\beta$ is the volumetric coefficient of thermal expansion. In the present work we assume that the micro-inertia density $j$ is constant. For the flow under study, it is relevant to assume that the applied magnetic field strength; $B(x)$ has the form

$B(x)=\frac{B_{0}}{\sqrt{x}}$,

where $B_{0}$ is a constant. Moreover, the electrical conductivity $\sigma_{0}^{\prime}$ is assumed to have the form:

$\sigma_{0}^{\prime}=\sigma_{0} u$, where $\sigma_{0}$ is a constant. The term $\frac{\sigma_{0}^{\prime}(B(x))^{2} u}{\rho}$ taking into account (6) and (7) can be written as:

$$
\frac{\sigma_{0}^{\prime}(B(x))^{2} u}{\rho}=\frac{\sigma_{0} B_{0}^{2} u^{2}}{\rho x}
$$

Using (1.8) the momentum equation (3) can be written as:

$u \frac{\partial u}{\partial x}+v \frac{\partial u}{\partial y}=\left(v+\frac{S}{\rho}\right) \frac{\partial^{2} u}{\partial y^{2}}+\frac{S}{\rho} \frac{\partial \sigma}{\partial y}+$

$g_{0} \beta\left(T-T_{\infty}\right) \operatorname{Cos} \alpha-\frac{\sigma_{0} B_{0}{ }^{2} u^{2}}{\rho x}$

Boundary conditions of the model are:

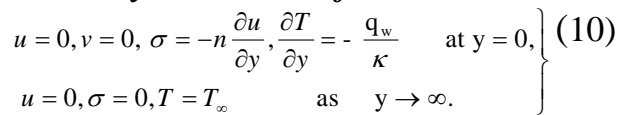

where the subscripts $w$ and $\infty$ refer to the wall and boundary layer edge, respectively.

\section{Non-dimensionalisation}

In order to obtain similarity solution of the problem we introduce the following nondimensional variables:

$$
\begin{aligned}
& \eta=y \sqrt{\frac{U_{0}}{2 v x}}, \psi=\sqrt{2 v U_{0} x} f(\eta), \\
& \sigma=\sqrt{\frac{U_{0}^{3}}{2 v x}} g, T-T_{\infty}=\theta(\eta) T^{*}
\end{aligned}
$$

where $\psi$ is the stream function, $U_{0}$ is some reference velocity and $T^{*}=\sqrt{\frac{2 v x}{U_{0}}}\left(\frac{q_{w}}{k}\right)$.Since $\quad u=\frac{\partial \psi}{\partial y} \quad$ and $v=-\frac{\partial \psi}{\partial x}$ we have from equation (11) that, $u=U_{0} f^{\prime}$ and $\quad v=-\sqrt{\frac{v U_{0}}{2 x}}\left(f-\eta f^{\prime}\right)$.

Here $f$ is non-dimensional stream function and prime denotes differentiation with respect to $\eta$.

Now substituting above equations into equations (9) and (4)-(5) we obtain,

$$
\begin{aligned}
& (1+\Delta) f^{\prime \prime \prime}+f f^{\prime \prime}+\Delta g^{\prime}+ \\
& \gamma \theta \operatorname{Cos} \alpha-M f^{\prime 2}=0, \\
& \left(1+\frac{1}{2} \Delta\right) \xi g^{\prime \prime}-2 \Delta\left(2 g+f^{\prime \prime}\right)+ \\
& \xi\left(f^{\prime} g+g^{\prime} f\right)=0, \\
& \theta^{\prime \prime}+\operatorname{Pr}\left(f \theta^{\prime}-f^{\prime} \theta\right)+\operatorname{Pr} Q \theta=0 .
\end{aligned}
$$


In the above equations we have used the following non-dimensional

parameters: $\Delta=\frac{S}{\mu}$ is the vortex viscosity parameter, $\xi=\frac{j U_{0}}{v x}$ is the micro-inertia density parameter, $\gamma=\frac{2 g_{0} \beta x T^{*}}{U_{0}^{2}}$ is the buoyancy parameter, $M=\frac{2 \sigma_{0} B_{0}^{2}}{\rho}$ is the magnetic field parameter, $\operatorname{Pr}=\frac{\mu c_{p}}{k}$ is the Prandtl number and $Q=\frac{2 Q_{0} x}{\rho c_{p} U_{0}}$ is the non dimensional heat generation parameter.

The corresponding boundary conditions (10) become,

$$
\left.\begin{array}{c}
f=f^{\prime}=0, g=-n f^{\prime \prime}, \theta^{\prime}=-1 \text { at } \eta=0, \\
f^{\prime}=0, g=0, \theta=0 \quad \text { as } \eta \rightarrow \infty .
\end{array}\right\}
$$

\section{Skin-Friction Coefficient, Plate Couple Stress and Nusselt Number}

The quantities of chief physical interest are the skin-friction coefficient, plate couple stress and the Nusselt number (rate of heat transfer). The local skin-friction coefficient is defined as

$$
C_{f}=\left(2 \operatorname{Re}_{x}^{-1}\right)^{\frac{1}{2}}[1+(1-n) \Delta] f^{\prime \prime}(0)
$$

Thus from equation (17) we see that the local values of the skin-friction coefficient $C_{f}$ is proportional to $f^{\prime \prime}(0)$.

The dimensionless couple stress is defined by

$$
M_{x}=\left(1+\frac{1}{2} \Delta\right) \xi g^{\prime}(0) \text {. }
$$

Thus the local plate couple stress in the boundary layer is proportional to $g^{\prime}(0)$.

Now the rate of heat transfer, in terms of the dimensionless Nusselt number, given by

$$
N u_{x}=\left(2^{-1} \operatorname{Re}_{x}\right)^{\frac{1}{2}} \frac{1}{\theta(0)} \text {. }
$$

Thus from equation (18) we see that the local Nusselt number $N u_{x}$ is proportional to $\frac{1}{\theta(0)}$.

\section{Method of Numerical Solution}

The set of nonlinear ordinary differential equations (12)-(14) with boundary conditions
(15) are non-linear and coupled. It is difficult to solve them analytically. Hence we adopt a procedure to obtain the solution numerically. Here we use the standard initial- value solver shooting method namely Nachtsheim-Swigert [24] shooting iteration technique (guessing the missing value) together with sixth order Runge-Kutta-Butcher initial value solver.

\section{Results and Discussion}

For the purpose of discussing the results, the numerical calculations are presented in the form of non-dimensional velocity, microrotation and temperature profiles. In the calculations the values of the vortex viscosity parameter $\Delta$, buoyancy parameter $\gamma$, Prandtl number $\operatorname{Pr}$, non-dimensional heat generation parameter $Q$, magnetic field parameter $M$, microinertia density parameter $\xi$, and microrotation parameter $n$ are chosen arbitrarily. Fig. 2(a) shows the effect of vortex viscosity parameter $\Delta$ on the velocity profiles. From here we see that velocity profile increases with the increase of $\Delta$. Fig. 2(b) shows the effect of $\Delta$ on the microrotation profiles. From this figure we see that microrotation increases very rapidly with the increase of the vortex viscosity parameter $\Delta$. It is also understood that as the vortex viscosity increases the rotation of the micropolar constituents gets induced in most part of the boundary layer except very close to the wall where kinematic viscosity dominates the flow. From Fig. 2(c) we found that the temperature profile increases for the increase of $\Delta$.

The effect of the angle of inclination $\alpha$ on the velocity field is shown in Fig. 3(a). From this figure we see that velocity decreases with the increase of $\alpha$. As $\alpha$ increases the effect of the buoyancy force decreases since it is multiplied by $\operatorname{Cos} \alpha$, so the velocity profile decreases. Fig. 3(b) shows the effect of $\alpha$ in the microrotation profiles. From this figure we observe that $\alpha$ has increasing effect on the microrotation profiles. Angle of inclination has also increasing effect on the temperature profiles that is shown in the Fig. 3(c).

Fig. 4(a) we see that velocity profiles increase with the increase of the microinertia density parameter $\xi$. The effect of $\xi$ is significant on the microrotation profiles. From Fig. 4(b) we see that microrotation decreases very rapidly with the increase of the microinertia density 
parameter. From Fig. 4(c) we see that the values of $\xi$ introduce slightly increasing effect on the temperature profiles.

Fig. 5(a) shows the free convection as well as the forced convection effects on the velocity profiles. This figure reveals that velocity increases as $\gamma$ increases. Fig. 5(b) shows the microrotation profiles for different values of $\gamma$. The angular velocity $g$ decreases with the increase of $\gamma$. It is also clear that very close

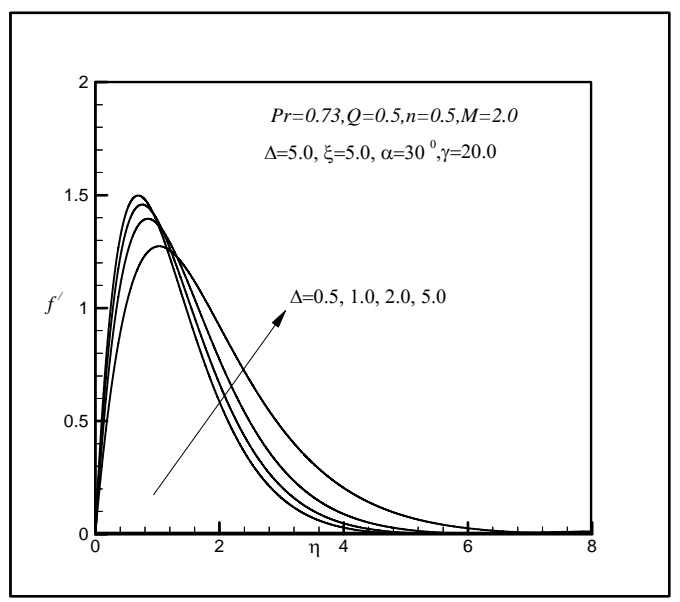

(a)

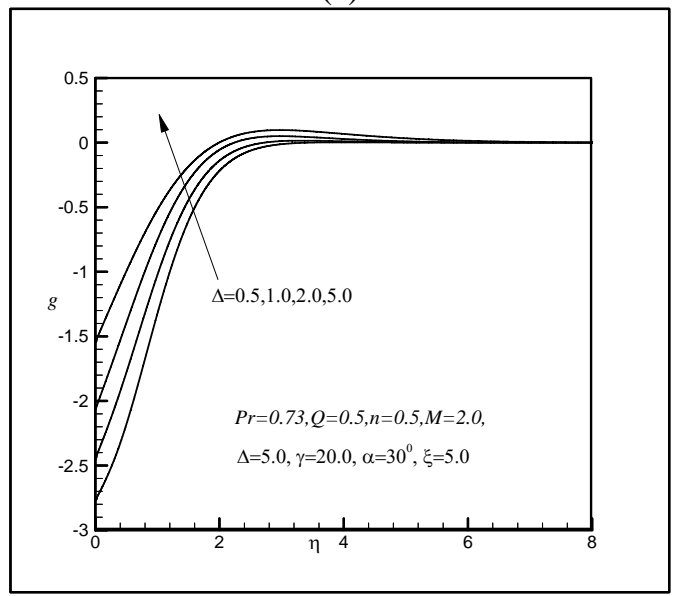

(b) to the plate these profiles remain negative and far away from it they become positive, whereas Rahman and Sattar [21] found that for purely forced convection as well as for mixed convection microrotation remains positive for a uniformly heated plate. Figure 5(c) shows the decreasing effect of $\gamma$ on the temperature profiles.

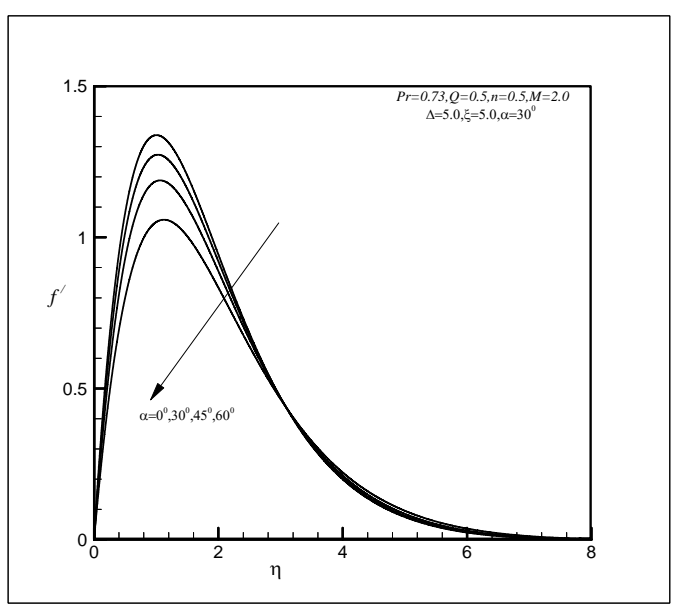

(a)

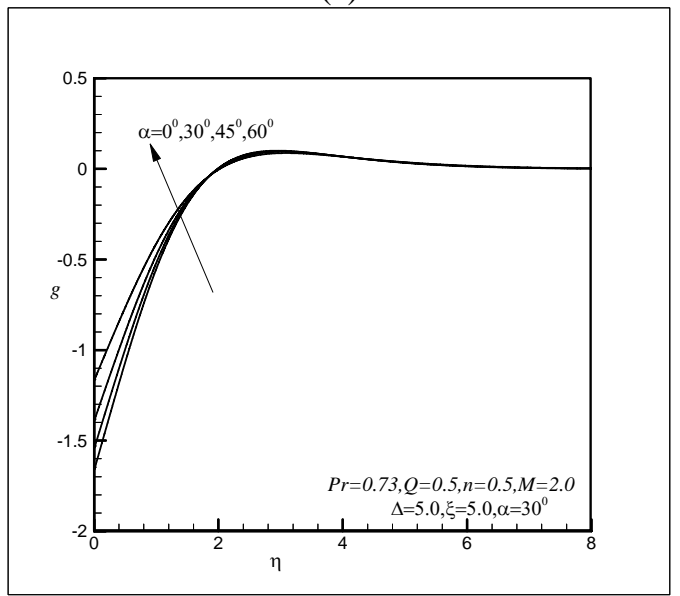

(b) 


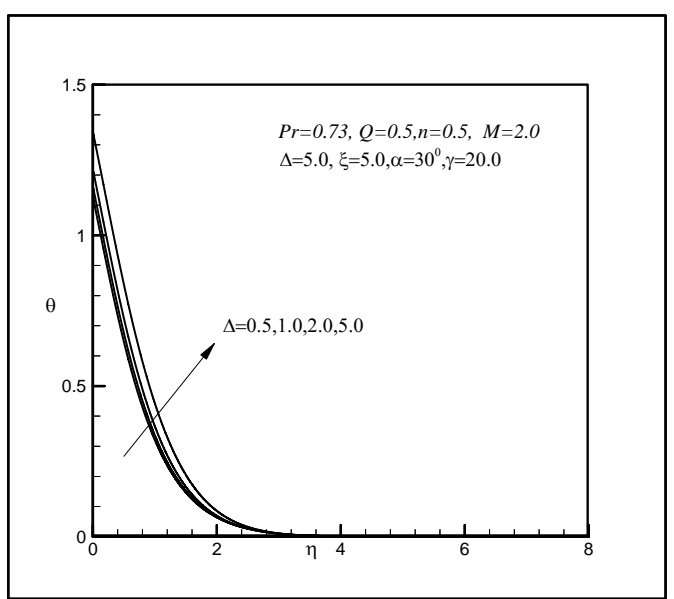

(c)

Fig. 2: Variations of non-dimensional (a) velocity, (b) microrotation and (c) temperature profiles for different values of $\Delta$.

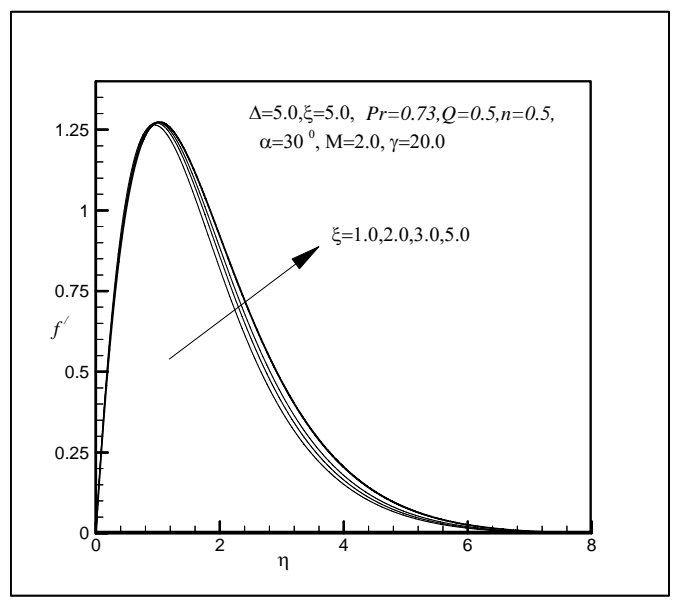

(a)

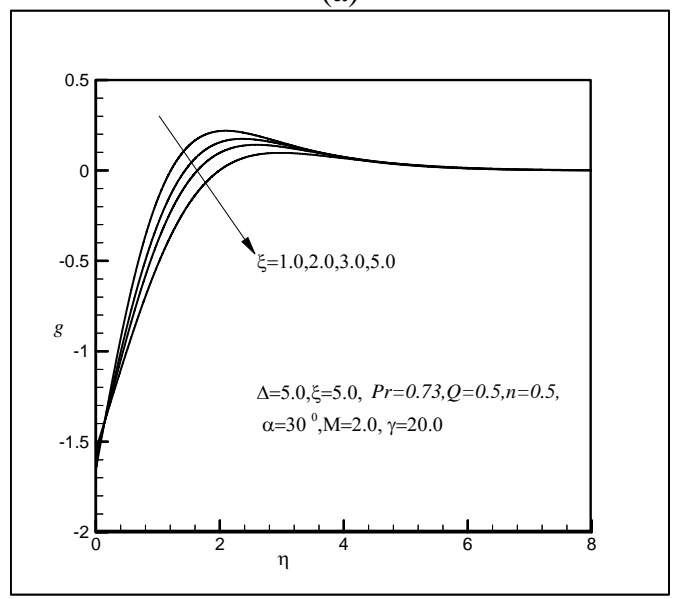

(b)

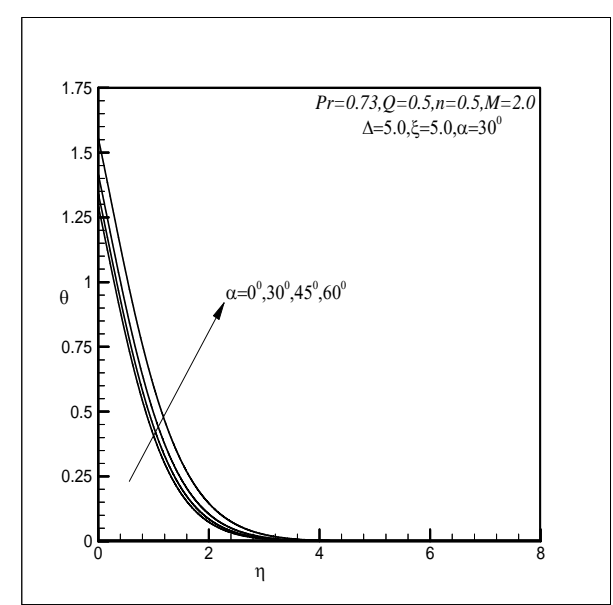

(c)

Fig. 3 Variations of non-dimensional (a) Velocity (b) Microrotation and (c) Temperature profiles for different values of $\alpha$.

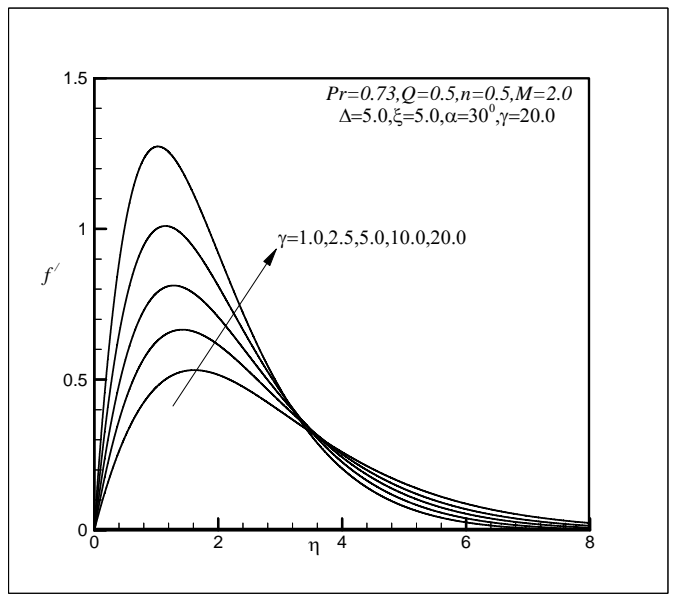

(a)

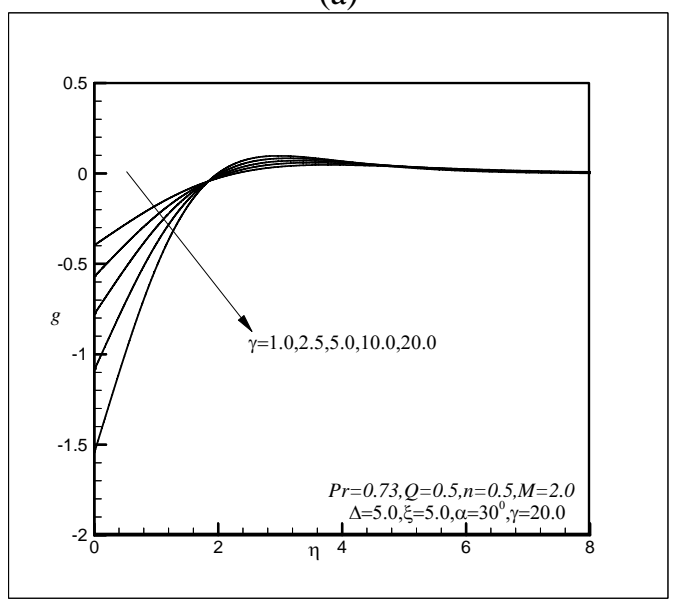

(b) 


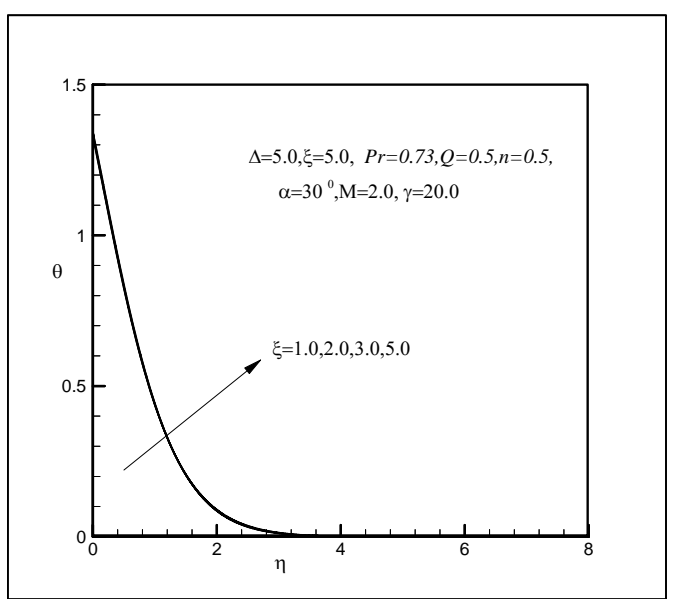

(c)

Fig. 4: Variations of non-dimensional (a) Velocity (b) Microrotation and (c) Temperature profiles for different values of $\xi$.

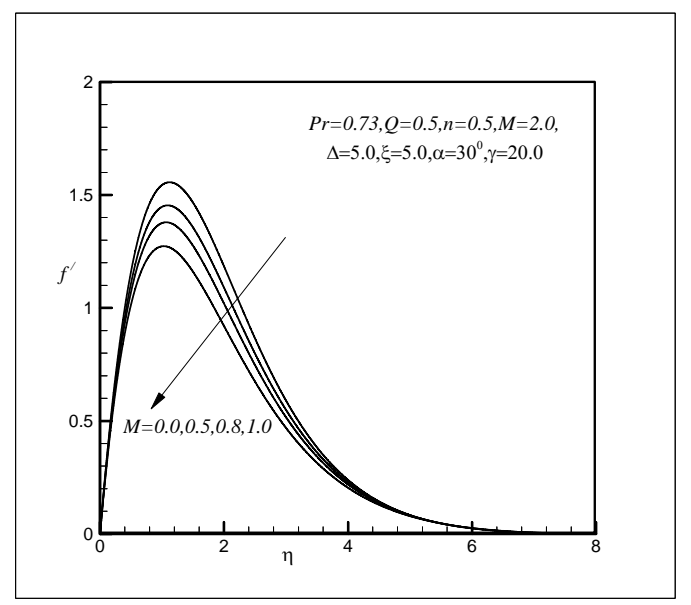

(a)

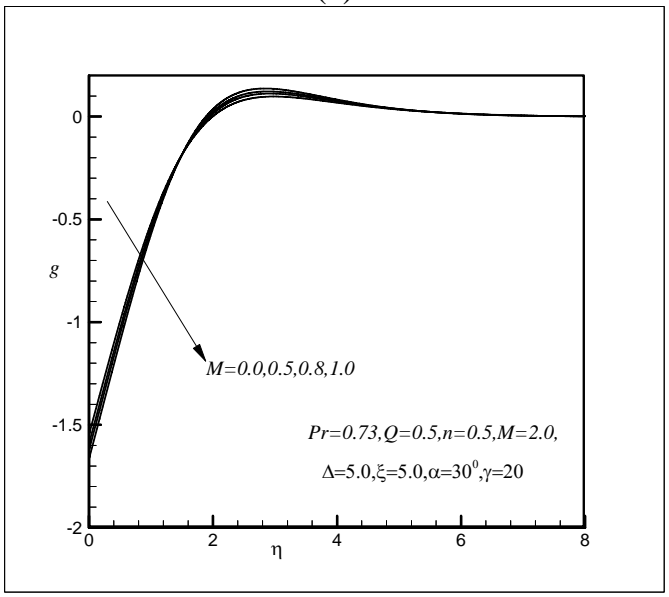

(b)

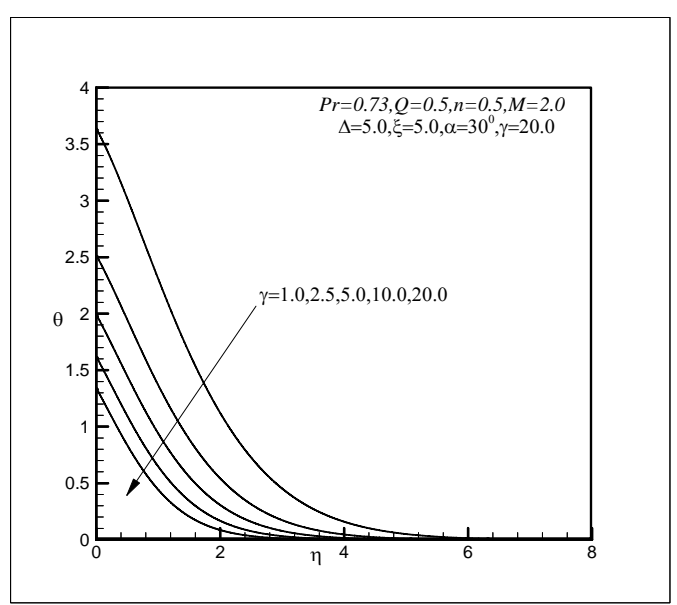

(c)

Fig. 5: Variations of non-dimensional (a) Velocity (b) Microrotation and (c) Temperature profiles for different values of $\gamma$.

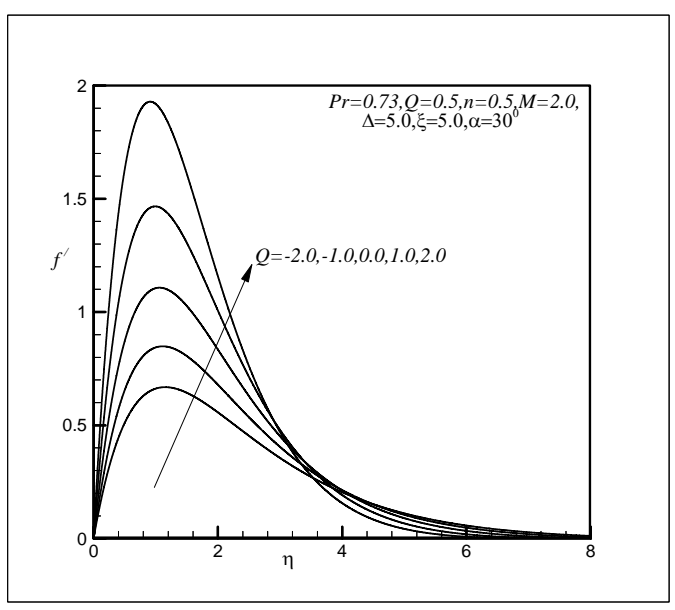

(a)

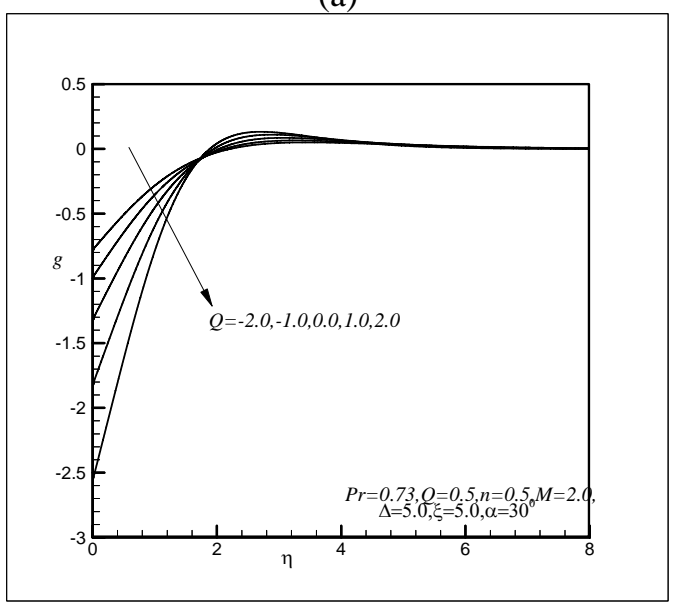

(b) 


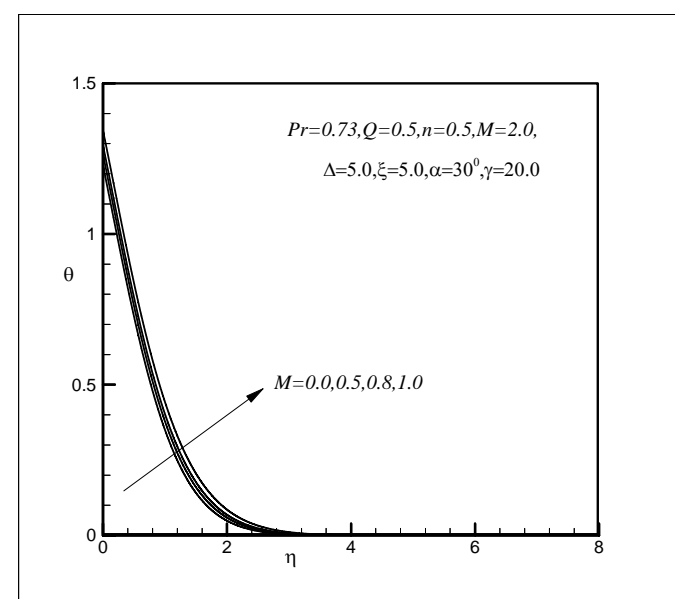

(c)

Fig. 6: Variations of non-dimensional (a) Velocity (b) Microrotation and (c) Temperature profiles for different values of $\mathrm{M}$.

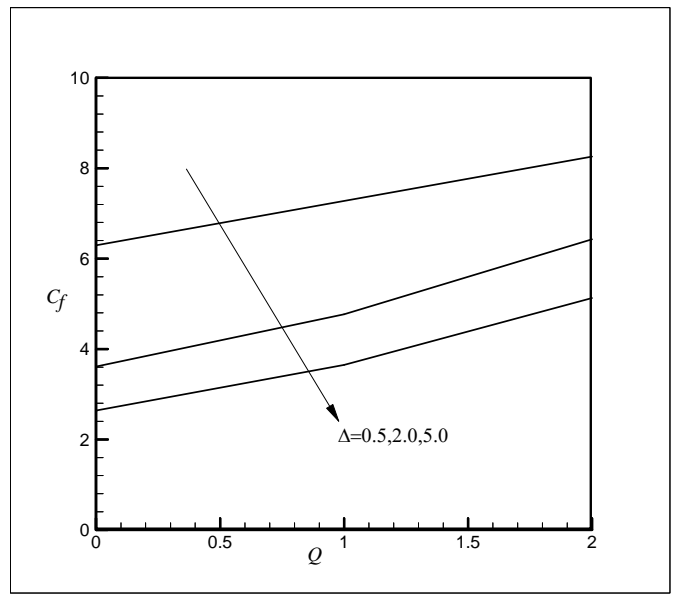

(a)

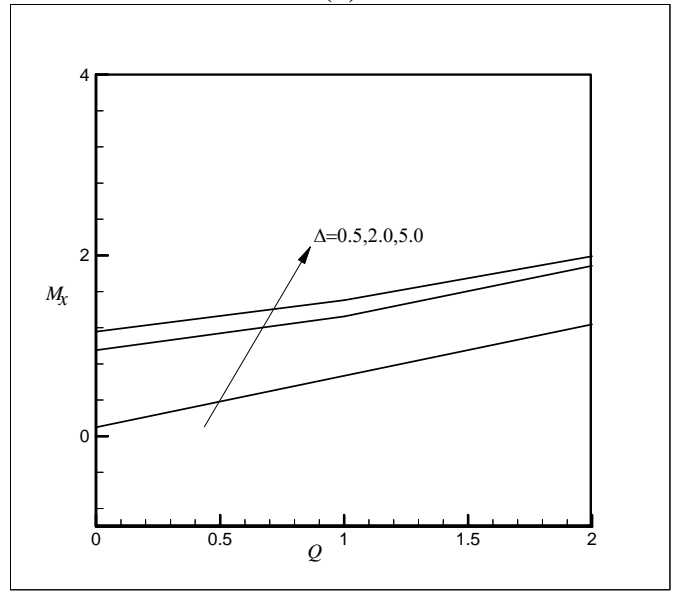

(b)

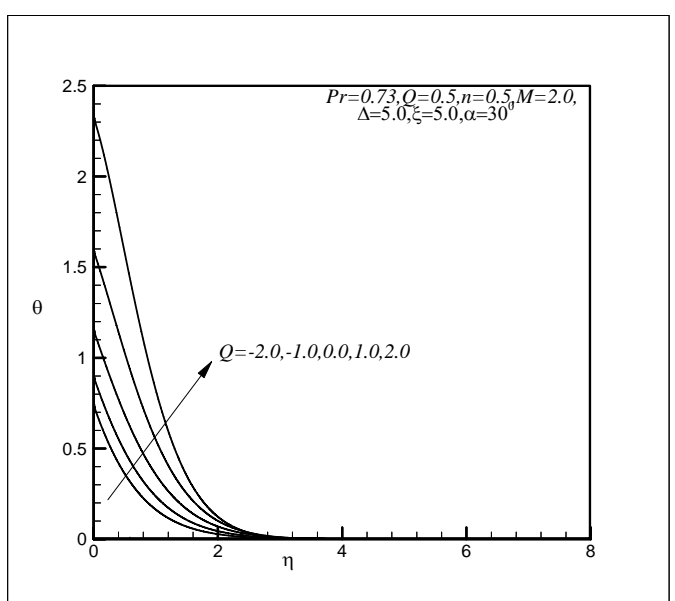

(c)

Fig. 7: Variations of non-dimensional (a) Velocity (b) Microrotation and (c) Temperature profiles for different values of $\mathrm{Q}$.

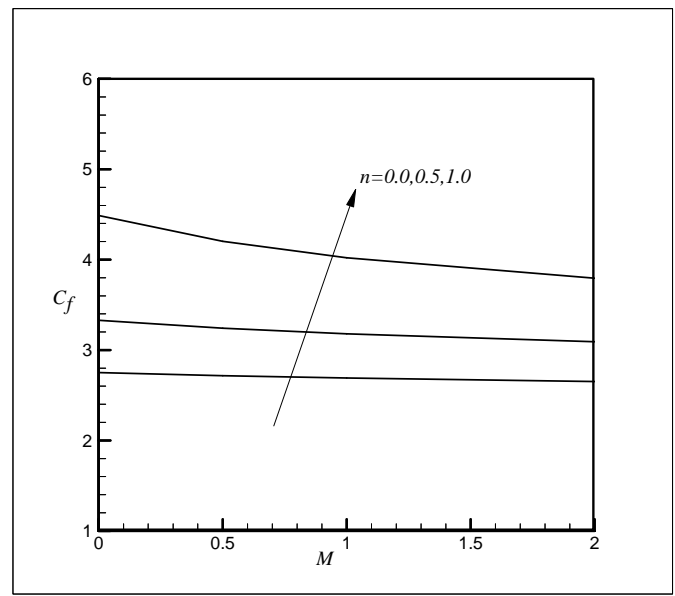

(a)

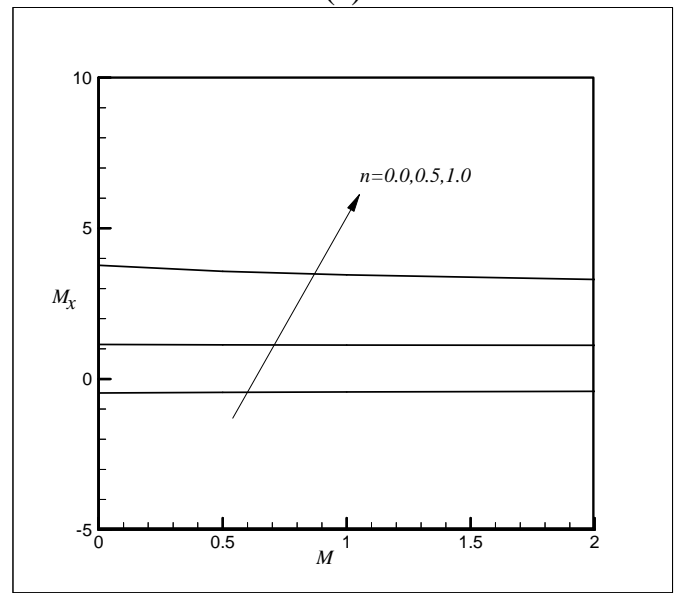

(b) 


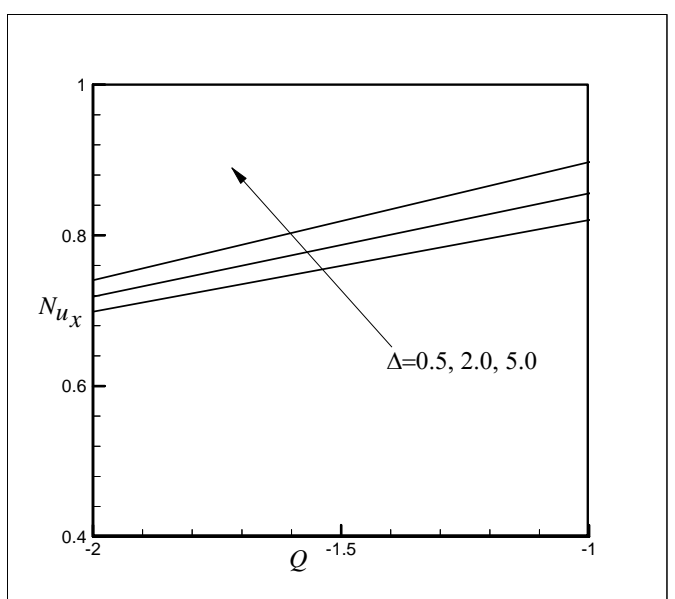

(c)

Fig. 8: Variations of (a) local skin-friction (b) rate of coupling and (c) Nusselt number for different values of $\Delta$ and $Q$.

From Fig. 6(a) we see that velocity profiles decrease with the increase of magnetic field parameter indicating that magnetic field tends to retard the motion of the fluid. These effects are much stronger near the surface of the plate. From Fig. 6(b) we see that microrotation profiles decrease with the increase of magnetic field parameter. Fig. 6(c) reveals that temperature profiles increase with the increase of $M$. This is due to the fact that the magnetic field tends to retard the velocity field, which in turn induces the temperature field resulting in an increase of the temperature profiles. The magnetic field can therefore be used to control the flow and heat transfer characteristics.

Fig. 7(a)-(c), respectively, shows the velocity, microrotation and temperature profiles for different values of heat generation/absorption parameter $Q$. Positive values of $Q$ represent the heat generation to the fluid and the negative values of $Q$ represent the heat absorption from the fluid. From Fig. 7(a) it is observed that when the heat is generated $(Q>0)$ the buoyancy force increases, which induce the flow rate to increase giving, rise to the increase in the velocity profiles. Again when the heat absorption $(Q<0)$ intensifies the velocity is found to decrease due to the decrease in the buoyancy force. Fig. 7(b) shows the decreasing effect of $Q$ on the microrotation profiles. Owing to the presence of heat generation it is apparent that there is an

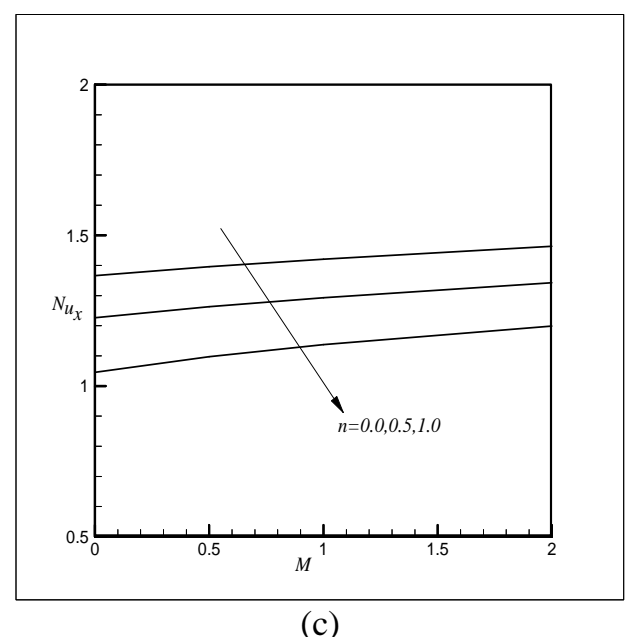

Fig. 9: Variations of (a) local-skin friction (b) rate of coupling and (c) Nusselt number for different values of $n$ and $M$.

increase in the thermal state of the fluid. Hence from Fig. 7(c), we observe that temperature increases as $Q$ increases.

In Fig. 8(a)-(c), respectively, we present the physical parameters skin-friction coefficient, plate couple stress and From Figure 8(a) we see that skin-friction $C_{f}$ decreases rapidly with the increase of $\Delta$ and increase with the increase of Q. Again from the Fig. 8(b)-(c) we see that the plate couple stress $M_{x}$ and Nusselt number $N u_{x}$ increases rapidly with the increase of $\Delta$ as well as with Q.

Fig. 9(a)-(c), respectively, shows that skinfriction coefficient, plate couple stress and the rate of heat transfer for different values of magnetic field parameter $M$ and the microrotation parameter $n$. Here we found skin-friction and plate couple stress increases with the increase of the magnetic field parameter $M$ whereas they decrease with the increase of the microrotation parameter $n$. From Fig. 9(c) we see that the rate of heat transfer decreases with the increase of the microrotation parameter $n$ while it increases with the increase of $M$. We also see that rate of heat transfer in a strongly concentrated micropolar fluid is higher than a weekly concentrated micropolar fluid.

\section{Conclusions}

Attention is focused on how the fluid velocity, angular velocity (microrotation) of the microconstituents and temperature 
profiles are affected by the variations of the vortex viscosity parameter $\Delta$, microinertia density parameter $\xi$, buoyancy parameter $\gamma$, magnetic field parameter $M$, heat generation parameter $Q$, and angle of inclination $\alpha$ and discussed them from the physical point of view. From the present calculation we may conclude that skin-friction coefficient (viscous drag) decreases monotonically with the increase of vortex viscosity parameter $\Delta$, magnetic field parameter $M$, Prandtl number $\operatorname{Pr}$, and angle of inclination $\alpha$. This coefficient increases with the increase of heat generation parameter $Q$ and microrotation parameter $n$. Plate couple stress increases with the increase of vortex viscosity parameter $\Delta$, heat generation parameter $Q$, and microrotation parameter $n$ while it decreases with the increase of magnetic field parameter $M$, Prandtl number Pr, and angle of inclination. The heat transfer rate increases with the increase of vortex viscosity parameter $\Delta$, heat generation parameter $Q$ and magnetic field parameter $M$. This rate of heat transfer is forced to decrease with the increase of Prandtl number $\operatorname{Pr}$, angle of inclination $\alpha$ and microrotation parameter $n$. In the case of heat generation $(Q>0)$ the thermal state of the fluid increases, consequently the heat transfer rate increases. For this case, the increase of the buoyancy force leads to the decrease of the flow rate in the boundary layer. The opposite phenomenon occurs for the heat absorption case.

\section{Acknowledge}

We would like to thanks the anonymous referees for their valuable comments which have incorporated to improve the paper.

\section{References}

[1] Ariman, T., Turk, M. A. and Sylvester, N. D. Microcontinuum fluid mechanics- a review, Intl. $J$. Eng. Sci., 12, 273-293, 1974.

[2] Hoyt, J. W. and Fabula, A. G. The effect of additives on fluid friction, US Naval Ordnance Test Station Report, 1964.

[3] Eringen, A. C., Theory of micropolar fluids, $J$. Math. Mech. 16, 1-18, 1966.

[4] Ebert, F. A similarity solution for the boundary layer flow of a polar fluid, Chem. Eng. J., 5, 8592, 1973.

[5] Jena, S. K. and Mathur, M. N., Similarity solution for laminar free convection flow of thermomicropolar fluid past a non-isothermal
[6] Soundalgekar, V. M. and Takhar, H. S. Flow of a micropolar fluid on a continuous moving plat, Intl. J. Eng. Sci. 21, 961, 1983.

[7] Gorla, R. S. R. and Takhar, H. S., Free convection boundary layer flow of a micropolar fluid past slender bodies, Intl. J. Engng. Sci., 25, 949-962, 1987.

[8] Yucel, A. Mixed convection micropolar fluid flow over horizontal plate with surface mass transfer, Intl. J. Engng. Sci., 27, 1593-1608, 1989.

[9] Gorla, R. S. R., Mixed convection in a micropolar fluid from a vertical surface with uniform heat flux, Intl. J. Engng. Sci. 30, 349-358, 1992

[10] Khonsari, M. M. and Brew, D. On the performance of finite journal bearing lubricated with micropolar fluids, ASLE Tribology Trans., 32, 155-160, 1989.

[11] Khonsari, M. M. On the self-excited whirl orbits of a journal in a sleeve bearing lubricated with micropolar fluids, Acta Mech., 81, 235-244, 1990.

[12] Gorla, R. S. R., Lin, P. P., and Yang, A. J. Asymptotic boundary layer solutions for mixed convection from a vertical surface in a micropolar fluid, Intl. J. Engng. Sci., 28, 525-533, 1990.

[13] Gorla, R. S. R., and Nakayam, S. Combined convection from a rotating cone to micropolar fluids, Math. Model. Sci. Compt., 25, 949-962, 1993.

[14] Alam M. S., Rahman, M. M. and Samad, M. A. Numerical study of the combined free-forced convection and mass transfer flow past a vertical porous plate in a porous medium with heat generation and thermal diffusion, Nonlinear Anal. Model. Control., 11, 331-343, 2006b.

[15] Alam, M. S., Rahman, M. M. and Sattar, M. A. Effects of thermophoresis and chemical reaction on unsteady hydro magnetic free convection and mass transfer flow past an impulsively started infinite inclined porous plate in the presence of heat generation/absorption, Thammasat Intl. J. Sci. Tech., 12, 44-52, 2007.

[16] Alam, M. S., Rahman, M. M. and Sattar, M. A. Effects of variable suction and thermophoresis on steady MHD combined free-forced convective heat and mass transfer flow a semi-infinite permeable inclined plat in the presence of thermal radiation, Intl. J. Thermal Sci., 2008 (in press, available online).

[17] Ariman, T., Turk, M. A. and Sylvester, N. D. Microcontinuum fluid mechanics- a review, Intl. $J$. Eng. Sci., 12, 273-293, 1974.

[18] Char, M. I. and Chang, C. L., Laminar free convection flow of micropolar fluids from a curved surface, J. Phys. D: Appl. Phys., 28, 13241331, 1995.

[19] Ebert, F. A similarity solution for the boundary layer flow of a polar fluid, Chem. Eng. J., 5, 8592, 1973.

[20] Eringen, A. C., Theory of micropolar fluids, $J$. Math. Mech. 16, 1-18, 1966.

[21] Gorla, R. S. R. and Takhar, H. S., Free convection boundary layer flow of a micropolar fluid past slender bodies, Intl. J. Engng. Sci., 25, 949-962, 1987. 
[22] Gorla, R. S. R., Mixed convection in a micropolar fluid from a vertical surface with uniform heat flux, Intl. J. Engng. Sci. 30, 349-358, 1992.

[23] Gorla, R. S. R., Lin, P. P., and Yang, A. J. Asymptotic boundary layer solutions for mixed convection from a vertical surface in a micropolar fluid, Intl. J. Engng. Sci., 28, 525-533, 1990.

[24] Gorla, R. S. R., and Nakayam, S. Combined convection from a rotating cone to micropolar fluids, Math. Model. Sci. Compt., 25, 949-962, 1993.

[25] Helmy, K. A, MHD boundary layer equations for power law fluids with variable electric conductivity, Mechanica, 30, 187-200, 1995.

[26] Hoyt, J. W. and Fabula, A. G. The effect of additives on fluid friction, US Naval Ordnance Test Station Report, 1964.

[27] Jena, S. K. and Mathur, M. N., Similarity solution for laminar free convection flow of thermomicropolar fluid past a non-isothermal vertical flat plate, Intl. J. Engng. Sci. 19, pp. 1431-39, 1981.

[28] Jones, D. R. Free convection from a semi-infinite flat plate inclined at a small angle to the horizontal, Quart. J. Mcch. Appl. Math., 128, 7798.1973.

[29] Khonsari, M. M. On the self-excited whirl orbits of a journal in a sleeve bearing lubricated with micropolar fluids, Acta Mech., 81, 235-244, 1990.

[30] Khonsari, M. M. and Brew, D. On the performance of finite journal bearing lubricated with micropolar fluids, ASLE Tribology Trans., 32, 155-160, 1989.

[31] Mohammadein, A. A. and Gorla, R. S. R., Heat transfer in a micropolar fluid over a stretching sheet with viscous dissipation and internal heat generation, Intl. J. Num. Meth. Heat Fluid Flow. 11(1), pp. 50-58, 2001.

[32] Molla, M. M., Hossain, M. A., and Yao, L. S. Natural convection flow along a vertical wavy surface with uniform surface temperature in presence of heat generation/absorption, Intl. J. Therm. Sci., 43, 157-163, 2004.

[33] Nachtsheim, P. R. and Swigert, P., Swigert, Satisfaction of the asymptotic boundary conditions in numerical solution of the system of non-linear equations of boundary layer type, NASSA TND3004, 1965.

[34] Power, H. Micropolar fluid model for the brain fluid dynamics, Intl. Conf. on bio-fluid mechanics, 1998, U.K.

[35] Rahman, M. M. and Sattar, M. A. MHD Free convection and mass transfer flow with oscillatory plate velocity and constant heat source in a rotating frame of reference, Dhaka Uni. J. Sci., 47, 63-73, 1999.

[36] Rahman, M. M. and Sattar, M. A. Magnetohydrodynamic convective flow of a micropolar fluid past a continuously moving vertical porous plate in the presence of heat generation/absorption, ASME J. Heat Trans., 128, 142-152, 2006.

[37] Rahman, M. M. and Sattar, M. A., Transient convective flow of micropolar fluid past a continuously moving vertical porous plate in the presence of radiation, Int. J. App. Math. Egng., 12, 497-513, 2007.

[38] Rahman, M. M. and Sultana, T. Convective flow of a micropolar fluid past a vertical porous plate immersed in a porous medium in the presence of radiation, J. Comput. Appl. Mecha. 2008b (accepted).

[39] Raptis, A. Flow of a micropolar fluid past a continuously moving plate by the presence of radiation, Intl. J. Heat Mass Transfer. 41, 28652866, 1998.

[40] Rees, D. A. S. and Pop, I. Free convection boundary layer flow of micropolar fluid from a vertical flat plat, IMA J. Appl. Math. 61, 179-97, 1998.

[41] Soundalgekar, V. M. and Takhar, H. S. Flow of a micropolar fluid on a continuous moving plat, Intl. J. Eng. Sci. 21, 961, 1983.

[42] Vajravelu, K. and Hadjinicolaou, A. Heat transfer in viscous fluid over a stretching sheet with viscous dissipation and internal heat generation, Intl. Commun. Heat Mass Transfer., 20, 417-430, 1993.

[43] Yucel, A. Mixed convection micropolar fluid flow over horizontal plate with surface mass transfer, Intl. J. Engng. Sci., 27, 1593-1608, 1989. 